\title{
AN ESTIMATION OF METEOROID FLUX AT OUTER MARTIAN SPACE FOR STEADY METEOR STREAMS
}

\author{
Ko NAGASAWA \\ Earthquake Research Institute, The University of Tokyo, \\ No. 1-1, Yayoi 1-chome, Bunkyo-ku, Tokyo, \\ JAPAN
}

\begin{abstract}
This work was done to estimate meteoroid fluxes of steady meteor streams at places far away from the sun, for example, beyond Martian orbit. For the estimation, a Normal Distribution Model is introduced. This model assumes that the flow of meteoroids is steady and the meteoroids are distributed in the form of a normal distribution around the orbit of their parent comet. Keplerian motion of each meteoroid is also assumed. Some parameters, necessary for determining a practical model, can be obtained through observations. The model will give the overall structure of meteor streams and their fluxes can be calculated for any points on the orbits of their parent comets. Calculations show that fluxes of streams sharply decrease as the solar distance increases and that meteoroids scatter chiefly along the orbital planes of the parent comets. The model seems to be useful to deduce rough structure of meteor streams.
\end{abstract}

\section{INTRODUCTION}

We have chances to see active meteor showers such as Perseids, Orionids and Geminids several times in a year. Some of these showers seem to show nearly the same activity every year and the flow of the meteoroids can be considered to be nearly steady in the solar system.

We can, however, observe the flow of meteoroids only on a line along the orbit of the earth. As a result, it is difficult to comprehend the whole structure of a meteor stream.

Direct measurements of the particle flux have often been tried using space probes. However, these measurements are restricted to particles of much smaller size. It is almost impossible for space probes to observe meteoroids of visual meteor size because their particle density in space is extremely low.

Then, in order to estimate meteoroid flux at places far away from the sun, in other words, to determine whole structure of a meteor stream, it is necessary to assume certain models for the distribution of meteoroid.

We introduce here a "Normal Distribution Model" for steady meteor streams. This model is based on the idea that meteoroid particles of a stream distribute nearly in the form of a normal distribution around the orbit of their parent comet. Though this simple assumption may differ from the real one, this model still gives some aspects to the meteoroid flux of steady streams in the solar system.

307

A.C. Levasseur-Regourd and H. Hasegawa (eds.), Origin and Evolution of Interplanetary Dust, 307-310.

(c) 1991 Kluwer Academic Publishers, Printed in Japan. 


\section{DETERMINATION OF MODEL PARAMETERS}

In order to specify the Normal Distribution Model, we have to define local coordinate systems. Take an arbitrary point $\mathbf{P}$ on the orbit of a parent comet and define a right-handed rectangular coordinate system $(u, v, w)$ with $P$ as its origin. Here, we take the positive direction of $v$-axis in the direction of the comet's motion and that of the $\mathrm{u}$-axis in the outward direction of the comet's orbit in the orbital plane. Then, the u-v plane coincides with the orbital plane of the comet. In this way, we can define a local coordinate system for any points on the orbit of the comet. Since the uw plane is always perpendicular to the flow of meteoroids, the number of meteoroids which pass through the plane per unit time gives the total flux of the stream. The positions where meteoroids pass through the plane give the distribution of the stream at $P$.

Let $P$ be the nearest point to the orbit of the earth, then a Normal Distribution Model is defined as follows:

(1) Each particle in the stream has Keplerian orbit.

(2) The flow of all meteoroids in the stream is steady.

(3) The positions where meteoroids pass through the $u$-w plane per unit time are two dimensional normal distribution with rexpect to (u.w) coordinates.

For applying the model to an actual meteor stream, total flux $\mathrm{N}$ and variances and covariance Suu, $\mathrm{Sww}$ and suw have to be determined as the model parameters. Assuming that the orbits of the parent comet and of the earth are straight and the meteoroid flow is uniform within the region where we observe the meteor shower, we can theoretically give the change in the meteor rate along the earth's orbit using the Normal Distribution Model. On the other hand, we can determine the earth's position $F$ where the shower is most active, the maximum flux $M$ and the variance $S$ for the duration of the shower from observations. Since the point $\mathrm{F}$ generally differs from $\mathrm{E}$, the nearest point to the comet's orbit, we can calculate the distance $\mathrm{L}$ between $\mathrm{E}$ and $\mathrm{F}$. We call $\mathrm{M}, \mathrm{S}$ and $\mathrm{L}$ observed parameters.

Model parameters can be calculated by comparing the observed parameters to those obtained from the theory. The model parameters are given as follows:

$$
\begin{aligned}
& \mathrm{S}_{\mathrm{uu}}=\alpha(\alpha \mathrm{W}-\gamma \mathrm{U}) \mathrm{S} /(\gamma \mathrm{L}+\mathrm{U}) \\
& \mathrm{S}_{\mathrm{WW}}=-\gamma(\alpha \mathrm{W}-\gamma \mathrm{U}) \mathrm{S} /(\alpha \mathrm{L}+\mathrm{W}) \\
& \mathrm{N}=2 \pi \mathrm{SM} \sqrt{\frac{\alpha \gamma(\alpha \mathrm{W}-\gamma \mathrm{U})^{2}}{(\alpha \mathrm{L}+\mathrm{U})(\gamma \mathrm{L}+\mathrm{W})}} \exp \left[-\frac{(\alpha \mathrm{L}+\mathrm{U})(\gamma \mathrm{L}+\mathrm{W})}{2 \alpha \gamma \mathrm{S}}\right]
\end{aligned}
$$

where $(\alpha, \beta, \gamma)$ are the direction cosines of the earth's orbit at $E$ and $(U, 0, W)$ are the coordinates of $\mathrm{E}$. Covariance suw is assumed to be zero because it is impossible to determine four model parameters from three observed parameters.

\section{FLUX CALCULATION}

In order to calculate a stream flux for an arbitrary point on the orbit of its parent comet, it is necessary to trace the entire path of each meteoroid in the stream. The Normal Distribution Model enables us to do the calculation.

This calculation corresponds, in short, to a transformation of the model parameters from a local system to another one. This includes, however, lengthy calculations and it is impossible to give all equation used. We show here only outline of the procedure.

Take a meteoroid particle which pass through the $u-w$ plane at positions $(u, 0, w)$ with 
velocity components $(\dot{\mathrm{u}}, \dot{\mathrm{V}}+\dot{\mathrm{v}}, \dot{\mathrm{w}}) . \dot{\mathrm{V}}$ is the velocity of the parent comet. We assume $u, w, \dot{\mathrm{u}}, \dot{\mathrm{v}}$ and $\dot{w}$ are small quantities and their quardratic terms can be neglected. Let the orbital elements of the comet be $(a, e, \Omega, i, \omega)$ and those of the particle be $(a+\Delta a, e+\Delta e, \Omega+\Delta \Omega, i+\Delta i, \omega+\Delta \omega)$. Then, $\Delta a$, $\Delta \mathrm{e}, \Delta \Omega, \Delta \mathrm{i}$ and $\Delta \omega$ can be expressed in linear forms of $\mathrm{u}, \mathrm{w}, \dot{\mathrm{u}}, \dot{\mathrm{v}}$ and $\dot{\mathrm{w}}$. This means that if $\Delta \mathrm{a}$, $\Delta \mathrm{e}, \Delta \mathrm{i}$ and $\Delta \omega$ are given the position coordinates $\left(\mathrm{u}^{\prime}, 0, \mathrm{w}^{\prime}\right)$ and velocity components $\left(\dot{\mathrm{u}}^{\prime}, \dot{\mathrm{V}}^{\prime}+\dot{\mathrm{v}}^{\prime}\right.$, $\left.\dot{w}^{\prime}\right)$ can be calculated for any other local system at $P^{\prime}$. This also means that we can calculate model parameters for any local system and that the meteoroid flux and the particle density are easily obtained from them.

\section{RESULTS}

We made the calculations for some permanent meteor streams. Though this shows us various characteristics of the meteor streams, some results are given in Table 1. In the calculations, orbital elements of the parent comets are taken from Marsden (1989) and those of the earth from Sidelman et al. (1974). Since the velocity distribution of each stream is difficult to obtain, we calculated it from the spread of the radiant. The spreads are from Millman (1963) and Koseki et al. (1990). Here, we assume the distribution of $(u, v, w)$ are isotropic and uniform for every point on the $\mathrm{u}-\mathrm{w}$ plane. Observed parameters are from Cook (1973).

Table1. Total flux, mean flux and particle density for three meteor streams at $1 \mathrm{AU}$ and $5 \mathrm{AU}$ from the sun.

\begin{tabular}{cllll}
\hline & $\eta$-Aquarids & Perseids & Lyrids & \\
\hline Total Flux & $1.2 \times 10^{13}$ & $7.6 \times 10^{10}$ & $7.4 \times 10^{9}$ & hour \\
\hline $\begin{array}{c}\text { Flux } \\
1 \mathrm{AU}\end{array}$ & $1.1 \times 10^{3}$ & 67 & 63 & $\left(10^{5} \mathrm{~km}^{2} \text { hour }\right)^{-1}$ \\
Density & 73 & 4.5 & 4.1 & $\left(10^{9} \mathrm{~km}^{3}\right)^{-1}$ \\
\hline $\begin{array}{c}\text { Flux } \\
\text { 5AU }\end{array}$ & 68 & 0.26 & $8.6 \times 10^{-3}$ & $\left(10^{5} \mathrm{~km}^{2} \text { hour }\right)^{-1}$ \\
Density & 11 & $4.1 \times 10^{-2}$ & $1.3 \times 10^{-3}$ & $\left(10^{9} \mathrm{~km}^{3}\right)^{-1}$ \\
\hline
\end{tabular}

\section{DISCUSSION AND CONCLUSIONS}

The largest problem in this estimation is to determine whether the Normal Distribution Model actually approximates the real distribution of meteoroids. For checking this, it is necessary to observe the flux in space. This seems to be difficult in the present stage of techniques. However, the following points are clarified about the structure of meteor streams through our calculations.

(1) The flux of meteoroids in a meteor stream shows extreme decrease as the solar distance increases.

(2) The meteoroids spread out mainly in the direction of orbital plane of heir parent comet.

(3) An erroneous choice in the radiant spread, that is, a wrong assumption in the velocity. distribution near the earth, doesn't introduce serious error in the flux of meteoroid at places far away from the sun.

(4) The determination of model parameters doesn't always give reasonable values in the case when the minimum distance between the orbits of the earth and the comet is large.

\section{REFERENCES}

Cook, A.F. (1973), 'A working list of meteor streams' in C.L. Hemenway, P.M. Millman and A.F. Cook( eds.) Evolutionary and Physical Properties of Meteoroids, NASA SP-319, 
pp.183-191.

Koseki, M., Sekiguchi, H. and Ohtsuka, K. (1990), 'Photographic Meteor Observations in Japan' in C.-I. Lagerkvist, H. Rickman, B.A. Lindblad and M. Lindgren (eds.) Asteroids, Comets, Meteors III, Uppsala, SWEDEN, pp.547-550.

Marsden, B.G. (1989), in Catalogue of Cometary Orbits, 6th edition, IAU Central Bureau for Astronomical Telegrams, Minor Planets Center.

Millman, P.M. and McKinley, D.W.R. (1963), 'Meteors' in B.M. Middlehurst and G.P. Kuiper (eds.) The Moon , Meteorites and Comets, The Solar System IV, The University of Chicago Press, U.S.A., pp.674-773.

Sidelmann, P.K., Doggett, L.E. and DeLuccia, M.R. (1974) 'Mean Elements of Principal Planets', A.J.79, pp.57-60. 